\title{
Optimising Large Animal Models of Sustained Atrial Fibrillation: Relevance of the Critical Mass Hypothesis
}

Nathan C. Denham ${ }^{*}$, Charles M. Pearman, George W. P. Madders, Charlotte E. R. Smith,
Andrew W. Trafford and Katharine M. Dibb

Unit of Cardiac Physiology, Institute of Cardiovascular Sciences, Manchester Academic Health Sciences Centre, The University of Manchester, Manchester, United Kingdom

Background: Large animal models play an important role in our understanding of the pathophysiology of atrial fibrillation (AF). Our aim was to determine whether prospectively collected baseline variables could predict the development of sustained AF in sheep, thereby reducing the number of animals required in future studies. Our hypothesis was that the relationship between atrial dimensions, refractory periods and conduction velocity (otherwise known as the critical mass hypothesis) could be used for the first time to predict the development of sustained AF.

Methods: Healthy adult Welsh mountain sheep underwent a baseline electrophysiology study followed by implantation of a neurostimulator connected via an endocardial pacing lead to the right atrial appendage. The device was programmed to deliver intermittent $50 \mathrm{~Hz}$ bursts of $30 \mathrm{~s}$ duration over an 8-week period whilst sheep were monitored for AF.

Results: Eighteen sheep completed the protocol, of which 28\% developed sustained AF. Logistic regression analysis showed only fibrillation number (calculated using the critical mass hypothesis as the left atrial diameter divided by the product of atrial conduction velocity and effective refractory period) was associated with an increased likelihood of developing sustained AF ( $\mathrm{Ln}$ Odds Ratio 26.1 [95\% confidence intervals 0.2-52.0] $p=0.048$ ). A receiver-operator characteristic curve showed this could be used to predict which sheep developed sustained AF (C-statistic 0.82 [95\% confidence intervals 0.59-1.04] $p=0.04$ ).

Conclusion: The critical mass hypothesis can be used to predict sustained AF in a tachypaced ovine model. These findings can be used to optimise the design of future studies involving large animals.

Keywords: atrial, fibrillation, critical, mass, hypothesis, pathophysiology, ovine, model

\section{INTRODUCTION}

Atrial fibrillation (AF) is a common arrhythmia characterised by rapid and disorganised atrial electrical activity, resulting in the loss of atrial contractility and an irregular ventricular response (Kirchhof et al., 2016). It is a major burden on healthcare systems worldwide as it is associated with an increased risk of cardiovascular morbidity (Wolf et al., 1991; Stewart et al., 2002) and 
mortality (Benjamin et al., 1998). A significant part of our current understanding of the underlying pathophysiology can be attributed to the use of large animal models in preclinical studies that aim to replicate the natural history of AF in humans by inducing progressive remodelling of the cardiac atria until a point where the arrhythmia is self-sustaining (Denham et al., 2018). Unfortunately, no model is perfect and it has been recognised that a subset of animals are resistant to developing sustained AF (Willems et al., 2001a). A guiding principle of animal research in the United Kingdom is summarised by the 3Rs (NC3Rs, 2020): replacement (methods to avoid or replace animal use), reduction (methods to minimise the number of animals used in studies) and refinement (methods to minimise suffering). Our aim was to prospectively collect baseline physiological variables known to influence the vulnerability to $\mathrm{AF}$ in humans and determine which (if any) improved the ability to predict the development of sustained $\mathrm{AF}$ in our ovine tachypacing model. This knowledge could then be applied to improve future experimental efficiency by reducing the overall number of animals required to produce sustained AF.

The current understanding of the pathophysiology of AF has been extensively reviewed elsewhere (Heijman et al., 2014; Denham et al., 2018). A key tenet is the critical mass hypothesis which states that: the probability of sustaining $\mathrm{AF}$ is proportional to the dimensions of the atrial tissue divided by the wavelength (the atrial effective refractory period times the conduction velocity) (Moe et al., 1964). In brief, AF is believed to be formed by multiple re-entrant wavelets of excitation whose optimal size is determined by the wavelength. The shorter the wavelength (generated by atria with a shorter effective refractory period and slower conduction velocity), the more wavelets can be sustained at the same time in a fixed volume of tissue reducing the likelihood of all extinguishing concurrently terminating AF (Moe et al., 1964). A larger atrial tissue dimension on the other hand will allow for more wavelets of a fixed size to be accommodated at the same time, reducing the likelihood all will terminate at a single time point. Our original hypothesis was that in a cohort of healthy sheep devoid of any pre-existing pathological remodelling, animals that could sustain the greatest number of re-entrant wavelets according to the critical mass hypothesis would be more likely to develop sustained AF during a tachypacing protocol.

\section{METHODS}

All procedures were in accord with The UK Animals (Scientific Procedures) Act (1986) and EU directive 2010/63. Institutional approval was received from The University of Manchester Animal Welfare and Ethical Review Board. Reporting follows the ARRIVE (Animal Research: Reporting of in vivo Experiments) guidelines (Kilkenny et al., 2010).

\section{Animal Model}

A total of 19 adult female Welsh mountain sheep of approximately 18 months of age were used in this study.
Only female sheep were included due to the limited availability of aged-matched males as a result of standard United Kingdom farming practices performing castration in the postnatal period. Sheep were group housed in a facility maintained on a 12-h light/12-h dark cycle, and afforded amble bedding and a diet of hay. All were free of observable disease and had acclimatised to the housing facility for a minimum of 1 week prior to starting the study. Data were included for all sheep that completed the pre-defined experimental protocol. One sheep was excluded from analysis who failed to complete the protocol due to displacement of the atrial pacing lead into the ventricle, requiring euthanasia as a humane end-point.

\section{Atrial Fibrillation Protocol}

Sheep were anaesthetised with $3 \% \mathrm{v} / \mathrm{v}$ isoflurane and right jugular venous access was performed via cut down and venotomy. Permanent pacing leads were advanced to the right ventricular apex and the right atrial appendage, after which they were connected to a dual chamber internal cardiac defibrillator (Medtronic, Woburn, MA). The defibrillator allowed for the wireless recording of atrial and ventricular electrograms in the experimental sheep, minimising any confounding effects of the stress associated with frequent handling on the inducibility of atrial fibrillation. A third pacing lead was placed in a separate location in the right atrial appendage and connected to a Medtronic Itrel-4 neurostimulator. The neurostimulator was programmed to deliver intermittent $50 \mathrm{~Hz}$ burst pacing in a repetitive cycle of $30 \mathrm{~s}$ on, $30 \mathrm{~s}$ off; at a minimum output of three times pacing threshold (range 3-5 V) with a pulse width of $390 \mathrm{~ms}$. The $30 \mathrm{~s}$ on/off cycle was chosen to allow for the quantification of AF duration during the off period on a daily basis, minimising the handling that would have been required to deactivate the neurostimulator if it had been programmed for continuous pacing. All leads were secured to the adjacent muscle with 2'0 silk sutures and the device generators were buried in separate subcutaneous pockets. The wound was closed in two layers with 2'0 Vicryl sutures and the sheep were recovered. Perioperative analgesia and antimicrobials were given in the form of subcutaneous meloxicam ( $1 \mathrm{mg} / \mathrm{kg}$ bodyweight; Boehringer Ingelheim, Ingelheim am Rheim, Germany) and intramuscular Betamox (15 mg/kg bodyweight; Norbrook Laboratories, Newry, United Kingdom), respectively.

After a minimum of 1 week recovery period, the neurostimulator was activated for a duration of 8 weeks. The 8 -week duration of the study was chosen based on unpublished pilot data from our group $(n=4)$, performed to establish the timeframe an intermittent pacing protocol would produce sustained AF in sheep. The daily burden of AF was monitored via wireless communication with the defibrillator, measuring the duration of AF during the $30 \mathrm{~s}$ off period. At the end of the 8-week protocol, the presence of sustained AF was documented, defined by deactivating the neurostimulator in the conscious sheep and recording $15 \mathrm{~min}$ of uninterrupted AF. At the end of the 8-week period, sheep were euthanised by permanent interruption of the circulation under terminal anaesthesia with 
pentobarbitone $(200 \mathrm{mg} / \mathrm{kg}$ bodyweight; AnimalCare, York, United Kingdom).

\section{Pre-operative Assessment}

Prior to surgery, bodyweight was recorded and the sheep in a casted position underwent a conscious transthoracic echocardiogram using a Vivid 7 ultrasound system (General Electric, Boston, MA). Left atrial anteroposterior diameter was measured in the parasternal long axis view as the mean over three cardiac cycles, as apical views could not be obtained in the sheep to assess left atrial volume. Epicardial fat pad thickness was measured anterior to the right ventricle in the parasternal long axis view using the atrioventricular groove as an anatomical reference, as per the method described by Iacobellis et al. (2003). An example echocardiographic image of measurements is provided in Supplementary Figure 1.

\section{Electrophysiology Studies}

A 5-lead electrocardiogram was recorded under general anaesthesia using Emka (Emka Technologies, Paris, France) and analysed in LabChart version 7 (AD instruments, Colorado Springs, CO). $P$ wave duration was taken as the maximum measurement recorded in any lead, and along with PR interval were averaged over 10 cycles in sinus rhythm prior to incision. Monophasic action potentials (MAPs) were recorded from the posterior wall of the right atrium using a Blazer electrophysiology catheter (Boston Scientific, Quincy, MA). Signals were digitised at $1 \mathrm{kHz}$ using a PowerLab amplifier and LabChart version 7 . The atrial effective refractory period (AERP) was determined by programmed electrical stimulation through the implanted defibrillator using a basic drive train of eight beats at $400 \mathrm{~ms}$ cycle length, followed by an extrastimulus starting at a coupling interval of $350 \mathrm{~ms}$ and decrementing in $10 \mathrm{~ms}$ intervals until the AERP was reached. The AERP was evidenced by the longest coupling interval which failed to produce a MAP, recorded using the Blazer electrophysiology catheter. The rate threshold for atrial MAP alternans was determined by delivering fixed burst atrial stimulation through the defibrillator over the cycle length range 500-250 ms. Alternans was assessed using 32-beat trains of action potentials and analysed using a spectral method as described previously (Pearman, 2014; Pearman et al., 2018), defining MAP alternans as trains with both a k-score $\geq 3$ and a $V_{\text {Alt }} \geq 0.02 \mathrm{mV}$. The rate threshold was the longest paced cycle length needed to produce atrial MAP alternans. The mean duration of induced AF was calculated after 10 bursts of $50 \mathrm{~Hz}$ noise at an output of $5 \mathrm{~V}$ for $5 \mathrm{~s}$. Atrial conduction velocity was recorded using a 2/8/2 decapolar electrophysiology catheter (Biosense Webster, Irvine, CA) placed on the lateral wall of the right atrium. Conduction velocity was calculated as the time taken to register a bipolar electrogram from the distal bipoles whilst pacing from the proximal bipoles via a Medtronic 2292 surgical pacing cable (Medtronic, Woburn, MA) attached to a Medtronic Carelink 2090 programmer. Right atrial conduction velocity was measured at the same cycle length as the AERP ( $400 \mathrm{~ms}$ ) as well as the maximum rate available through the stimulator (cycle length $273 \mathrm{~ms}$ ) in order to better approximate the rapid atrial electrical activity expected in AF.

\section{Data Analysis and Statistics}

Atrial wavelength was calculated using Equation 1, utilising conduction velocity measured at a paced cycle length of 400 and $273 \mathrm{~ms}$. The relationship of left atrial size to wavelength (hereto described as fibrillation number) was calculated using Equation 2 which was adapted from Hwang et al. (2015).

$$
\begin{aligned}
\text { Wavelength }(\mathrm{cm})= & A E R P(\mathrm{~ms}) X \text { Atrial conduction } \\
& \text { velocity }\left(\mathrm{cm} \cdot \mathrm{ms}^{-1}\right)
\end{aligned}
$$

Fibrillation number $=\frac{\text { Left atrial anteroposterior diameter }(\mathrm{cm})}{\text { Wavelength }(\mathrm{cm})}$

Raw data are reported as mean \pm standard error of the mean (SEM) and logistic regression data as natural logarithms of odds ratio (Ln OR) with the $95 \%$ confidence intervals (95\%CI). Statistical testing and presentation of continuous variables, logistic regression and receiver-operator characteristic (ROC) curve analysis was performed using Sigmaplot V11 (Systat Software, San Jose, CA). A two-tailed Student's $t$-test was used to compare continuous data where differences were considered significant when $p<0.05$. Logistic regression confidence intervals which crossed zero where considered non-significant. ROC curve analysis was considered non-significant where the $95 \% \mathrm{CI}$ of the C-statistic crossed 0.5. Optimal cut-off valves were determined from ROC curves with significant predictive value by calculating the Youden J statistic (Equation 3), where sensitivity and specificity are expressed numerically between zero and one. The Youden J statistic is a dimensionless index between zero and one, where a score of zero means the independent variable is no better a predictor than random chance, whereas a score of one represents a perfect predictor of sustained AF.

$$
\text { Youden J statistic }=(\text { Sensitivity }+ \text { Specificity })-1
$$

\section{RESULTS}

Eighteen sheep completed the 8-week pacing protocol, of which 5/18 (28\%) developed sustained AF. The remaining 13/18 (72\%) sheep were unable to sustain 15 min of uninterrupted AF and were classified as resistant. All 18 sheep underwent the baseline in vivo assessment of pre-specified variables, however, the rate threshold for atrial MAP alternans could only be determined in $15 / 18(83 \%)$.

\section{Sheep Who Developed Sustained Atrial Fibrillation Have a Larger Fibrillation Number at Baseline}

An overview of the baseline characteristics between the sustained $\mathrm{AF}$ and resistant cohort can be observed in Table 1. Variability in the characteristics between sheep in the absence of pre-existing 
TABLE 1 | Differences in baseline characteristics between the sheep developing sustained atrial fibrillation $(N=5)$ and those who were resistant $(N=13)$.

\begin{tabular}{lccc}
\hline Single variables (units) & Sustained AF & Resistant & Significance \\
\hline$P$ wave duration (ms) & $50.6 \pm 2.8$ & $45.7 \pm 2.9$ & $P=0.33$ \\
PR interval (ms) & $109.6 \pm 6.3$ & $109.6 \pm 2.4$ & $P=0.99$ \\
AERP (ms) & $132 \pm 7$ & $155 \pm 6$ & $P=0.05$ \\
Mean duration of induced AF (s) & $35.2 \pm 29.0$ & $6.4 \pm 3.4$ & $P=0.13$ \\
Weight (kg) & $37.8 \pm 2.1$ & $36.2 \pm 1.3$ & $P=0.53$ \\
Left atrial diameter (cm) & $2.62 \pm 0.10$ & $2.46 \pm 0.05$ & $P=0.11$ \\
Epicardial fat pad thickness (cm) & $0.70 \pm 0.11$ & $0.63 \pm 0.07$ & $P=0.60$ \\
Conduction velocity (CL400 ms) (m/s) & $0.93 \pm 0.09$ & $0.82 \pm 0.04$ & $P=0.27$ \\
Conduction velocity (CL273 ms) (m/s) & $0.76 \pm 0.08$ & $0.83 \pm 0.05$ & $P=0.51$ \\
Rate threshold atrial alternans (ms) & $308 \pm 9$ & $313 \pm 8$ & $P=0.71$ \\
Combined variables & & & \\
Wavelength (CL 400 ms) (cm) & $12.0 \pm 0.85$ & $12.6 \pm 0.54$ & $P=0.59$ \\
Wavelength (CL 273 $\mathbf{~ m s ) ~ ( c m ) ~}$ & $\mathbf{9 . 9} \pm \mathbf{0 . 8}$ & $\mathbf{1 2 . 7} \pm \mathbf{0 . 7}$ & $\boldsymbol{P}=\mathbf{0 . 0 4}$ \\
Fibrillation number (CL400 ms) & $0.22 \pm 0.02$ & $0.20 \pm 0.01$ & $P=0.32$ \\
Fibrillation number (CL273 $\mathbf{~ m s ) ~}$ & $\mathbf{0 . 2 7} \pm \mathbf{0 . 0 3}$ & $\mathbf{0 . 2 0} \pm \mathbf{0 . 0 1}$ & $\boldsymbol{P}=\mathbf{0 . 0 2}$ \\
\hline
\end{tabular}

Data presented as mean \pm SEM; statistical comparisons using an unpaired t-test; statistical significance taken as $p<0.05$. AERP, atrial effective refractory period; $A F$, atrial fibrillation; EFT, epicardial fat pad thickness; CL, paced cycle length.

disease reflects genetic variation in an outbred population. No significant differences between the groups were seen when looking at single variables alone, although the AERP trended toward being shorter in those who developed sustained $\mathrm{AF}$ $(132 \pm 7 \mathrm{~ms}$ vs. $155 \pm 6 \mathrm{~ms} ; p=0.05)$. When variables were combined according to the critical mass hypothesis to produce wavelength and fibrillation number, no difference was observed at a paced cycle length of $400 \mathrm{~ms}$ (wavelength: $12.0 \pm 0.85 \mathrm{~cm}$ vs. $12.6 \pm 0.54 \mathrm{~cm}, p=0.59$; fibrillation number: $0.22 \pm 0.02$ vs. $0.20 \pm 0.01, p=0.32$ ). In contrast, when wavelength and fibrillation number were recalculated at the shortest paced cycle length of $273 \mathrm{~ms}$, both a shorter wavelength $(9.9 \pm 0.8 \mathrm{~cm}$ vs. $12.7 \pm 0.7 \mathrm{~cm} ; p=0.04)$ and a larger fibrillation number $(0.27 \pm 0.03$ vs. $0.20 \pm 0.01$; $p=0.02)$ was observed in the group developing sustained AF. According to the critical mass hypothesis, this suggests naive sheep who go on to develop sustained AF are capable of sustaining a greater number of re-entrant fibrillatory wavelets for a fixed volume of atrial tissue, lowering the probability of $\mathrm{AF}$ terminating at a single point in time. As the AF cohort was small $(n=5)$, it was not possible to determine which variable (shorter AERP, slower conduction velocity or larger left atrial diameter at baseline) had the greatest influence on the larger fibrillation number.

\section{A Larger Fibrillation Number Increases the Probability of Developing Sustained AF}

We next asked whether any of the aforementioned variables could increase the probability of developing AF. A logistic regression analysis showed none of the single baseline variables were associated with an increased likelihood of developing sustained AF (Table 2). When the combined variables of wavelength
TABLE 2 | Univariate logistic regression analysis of single baseline characteristics between the sheep who develop sustained $\mathrm{AF}$ and those who were resistant.

\begin{tabular}{lcc}
\hline Variable (units) & Ln OR & $\mathbf{9 5 \% ~ C l}$ \\
\hline$P$ wave duration (ms) & 0.06 & -0.05 to 0.18 \\
PR interval (ms) & 0 & -0.11 to 0.11 \\
AERP (ms) & -0.05 & -0.11 to 0.01 \\
Mean duration of induced AF (s) & 0.03 & -0.02 to 0.07 \\
Weight (kg) & 0.09 & -0.17 to 0.34 \\
Left atrial diameter (cm) & 6.25 & -1.74 to 14.24 \\
Epicardial fat pad thickness (cm) & 1.15 & -2.94 to 5.25 \\
Conduction velocity (CL400 ms) (m/s) & 3.56 & -2.71 to 9.84 \\
Conduction velocity (CL273 ms) (m/s) & -2.10 & -8.07 to 3.87 \\
Rate threshold atrial alternans (ms) & -0.01 & -0.06 to 0.04 \\
Wavelength (CL 400 ms) (cm) & -17.0 & -74.43 to 40.40 \\
Wavelength (CL 273 ms) (cm) & -64.18 & -134.74 to 6.39 \\
Fibrillation number (CL400 ms) & 13.83 & -12.59 to 40.25 \\
Fibrillation number (CL273 ms) & 26.12 & 0.22 to 52.01 \\
\hline
\end{tabular}

Data presented as natural logarithms of odds ratio ( $L n O R$ ) with 95\% confidence intervals (Cl). 95\% Cl which cross zero are non-significant. AERP, atrial effective refractory period; $C L$, paced cycle length.

and fibrillation number were analysed, wavelength was not associated with an increased likelihood of developing sustained $\mathrm{AF}$ at either a paced cycle length of $400 \mathrm{~ms}$ (Ln OR -17.0 [95\%CI -74.4 to 40.4$]$ ) or $273 \mathrm{~ms}$ ( $\mathrm{Ln} \mathrm{OR}-64.2$ [95\%CI -134.7 to 6.4$]$ ). In contrast, fibrillation number was associated with an increased likelihood of developing sustained AF at a paced cycle length of $273 \mathrm{~ms}$ (Ln OR 26.1 [95\%CI 0.252.0] $p=0.048)$ but not at a paced cycle length of $400 \mathrm{~ms}$ (Ln OR 13.8 [95\%CI -12.6 to 40.2$]$ ). In the context of the critical mass hypothesis, this means the larger the fibrillation number at baseline, the greater the likelihood of developing sustained AF.

\section{Fibrillation Number Can Be Used to Predict the Development of Sustained AF}

A receiver-operator characteristic (ROC) curve was created for fibrillation number calculated using a paced cycle length of $273 \mathrm{~ms}$ (Figure 1). This generated a C-statistic of 0.82 (95\% CI $0.59-1.04, p=0.04)$ demonstrating it is therefore possible to predict the likelihood of a sheep developing sustained $\mathrm{AF}$ using baseline variables prior to any pathological remodelling of the atria. To maximise the predictive power of the curve, the optimal cut-offs were calculated. The highest Youden $J$ statistic was attributed to a fibrillation number $>0.28$ (sensitivity $60 \%$, specificity $92 \%, J=0.52$ ). In summary, this suggests sustained $\mathrm{AF}$ had a greater likelihood of being observed in sheep with a combination of a larger left atrial diameter, a slower atrial conduction velocity measured at a paced cycle length of $273 \mathrm{~ms}$ and a shorter AERP. As such, although these parameters alone did not influence the likelihood of sustained AF, they could collectively be positively selected for to minimise the number of experimental animals who are resistant to developing sustained AF. 


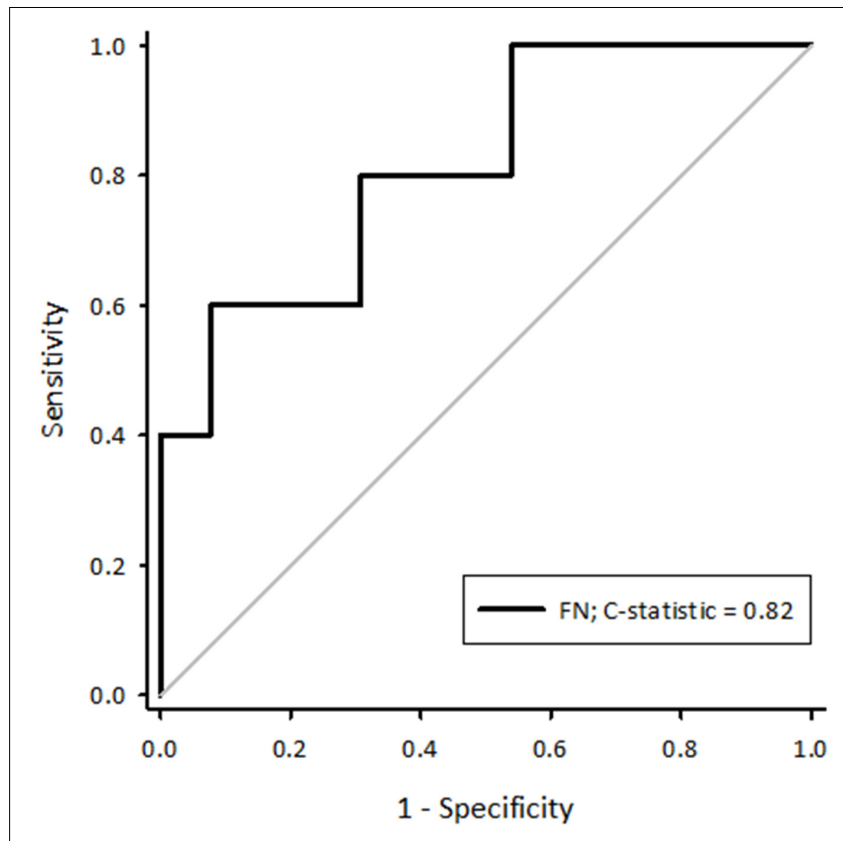

FIGURE 1 | A receiver-operator characteristic curve generated for fibrillation number calculated using a paced cycle length of $273 \mathrm{~ms}$. The C-statistic was 0.82 (95\% Cl 0.59-1.04, $p=0.04)$. FN, fibrillation number.

\section{DISCUSSION}

\section{Relevance of the Critical Mass Hypothesis to Developing Sustained AF in Tachypaced Sheep}

In this study, only fibrillation number was shown to be suitably predictive of sustained AF in sheep who underwent our 8-week tachypacing protocol. None of the constituent variables (atrial diameter, conduction velocity and effective refractory period) or the wavelength were significantly useful alone in predicting sustained AF. This supports our original hypothesis that it is the combination of the three variables which underpins the critical mass hypothesis and best predicts the likely experimental outcome. The critical mass hypothesis has previously been used to predict the duration of AF induced acutely in animals over a short timeframe of pacing [minute to hours (Byrd et al., 2005; Lee et al., 2013)] and also in humans with longstanding AF with significant atrial remodelling to predict treatment success (Hwang et al., 2015; Williams et al., 2019). As such, this is the first study to use the critical mass hypothesis to aid the prediction of sustained AF in long term animal models who also have no known pre-existing pathological atrial remodelling. This finding would support the critical mass hypothesis as part of the key mechanism underpinning the pathophysiology of $\mathrm{AF}$, which currently remains incompletely understood particularly what the basic re-entrant wavelet in AF represents (Nattel et al., 2017).

\section{Fibrillation Number Can Be Used to Improve Experimental Efficiency in Our Model}

Only $28 \%$ of sheep developed sustained AF during the experimental protocol. In line with the $3 \mathrm{Rs}$ of animal experimentation (NC3Rs, 2020), there is clearly room to improve experimental efficiency by reducing the number of resistant animals who ultimately go through the surgery and experimental protocol without reaching the desired endpoint. Our study has shown fibrillation number can be used to assist in predicting which animals are likely to develop sustained AF, however, it should be noted fibrillation number can only be calculated after an electrophysiology study. As such, sheep would need to undergo an invasive procedure prior to calculating their likelihood of developing sustained AF. In this situation, it is suggested animals with a low probability of developing sustained $\mathrm{AF}$ could be triaged to alternative experimental studies rather than embark upon an 8-week protocol which has a low likelihood of creating the desired endpoint (NC3Rs, 2020).

\section{Wider Implications for Ovine Models of Atrial Fibrillation}

A number of experimental AF protocols have been described in the literature, the commonest based upon rapid atrial pacing (Willems et al., 2001a,b; Anne et al., 2007; Pruvot et al., 2007; Lenaerts et al., 2009, 2013; Filgueiras-Rama et al., 2012; Martins et al., 2014; Monigatti-Tenkorang et al., 2014; Macquaide et al., 2015; Takemoto et al., 2016, 2017; Haemers et al., 2017) although methods involving autonomic modulation (Geddes et al., 1996) or structural insults (Deroubaix et al., 2004) have been described. At present, there is no protocol which is considered the gold standard for producing sustained AF in sheep. A summary of the reported methodologies relevant to tachypaced ovine models of sustained AF is produced in Table 3.

Key differences between tachypaced protocols can be categorised by: the nature of pacing (the stimulation frequency and whether it is delivered continuously or intermittently); the site of stimulation (right or left atrium); and the duration of pacing (range: 8 weeks to 6 months). These have been reported to have much better success rates (between 29 and 100\%) than seen in our study (Willems et al., 2001a,b; Anne et al., 2007; Pruvot et al., 2007; Lenaerts et al., 2009, 2013; Filgueiras-Rama et al., 2012; Martins et al., 2014; Monigatti-Tenkorang et al., 2014; Macquaide et al., 2015; Takemoto et al., 2016, 2017; Haemers et al., 2017), however, there is no way of accounting for potential underreporting of alternative protocols with higher failure rates. None of these reported protocols exactly matches our own, which we designed specifically to remotely monitor the daily inducibility of AF after intermittent pacing to study the influences on progressive atrial remodelling. Only a single study by Willems et al. (2001a) has directly compared two different tachypacing protocols between one based on continuous stimulation (right atrial pacing at cycle length $100 \mathrm{~ms}$ ) vs. one based on intermittent stimulation ( $2.5 \mathrm{~s}$ right atrial pacing at cycle length $24 \mathrm{~ms}$, whenever sinus rhythm was detected). Although neither methodology exactly matched our 
TABLE 3 | A summary of studies in the literature utilising a tachypacing methodology to induce sustained atrial fibrillation in sheep.

\begin{tabular}{|c|c|c|c|}
\hline $\begin{array}{l}\text { Tachypaced AF protocol: FIRST } \\
\text { author and references }\end{array}$ & Methodology of tachypacing & Defintion of sustained AF & $\begin{array}{c}\text { Success rate (\% sheep developing } \\
\text { sustained AF by the end of the } \\
\text { protocol) }\end{array}$ \\
\hline $\begin{array}{l}\text { Willems et al. (2001a,b), Anne et al. } \\
\text { (2007) }\end{array}$ & $\begin{array}{l}\text { Continuous right atrial pacing at cycle } \\
\text { length } 67 \text { ms for a mean of } \sim 6 \text { months }\end{array}$ & $\begin{array}{l}\text { AF lasting }>1 \mathrm{~h} \text { after cessation of } \\
\text { pacing }\end{array}$ & $70-77 \%$ \\
\hline $\begin{array}{l}\text { Pruvot et al. (2007), } \\
\text { Monigatti-Tenkorang et al. (2014) }\end{array}$ & $\begin{array}{l}\text { Intermittent right atrial pacing at cycle } \\
\text { length } 170 \text { ms for } 8 \text { weeks. Stepwise } \\
\text { protocol starting } 2 \text { s on } / 5 \text { s offuntil } \\
\text { maximum } 5 \text { s on } / 2 \text { s off }\end{array}$ & $\begin{array}{l}\text { AF lasting }>1 \text { week after cessation of } \\
\text { pacing }\end{array}$ & $100 \%$ \\
\hline $\begin{array}{l}\text { Lenaerts et al. (2009, 2013), Macquaide } \\
\text { et al. (2015), Haemers et al. (2017) }\end{array}$ & $\begin{array}{l}\text { Continuous right atrial pacing at cycle } \\
\text { length } 100 \text { ms for } 15 \text { weeks }\end{array}$ & $\begin{array}{l}\text { Absence of sinus rhythm after } \\
\text { cessation of pacing for the remaining } \\
\text { follow up period until euthanasia }\end{array}$ & $100 \%$ \\
\hline $\begin{array}{l}\text { Filgueiras-Rama et al. (2012), Martins } \\
\text { et al. (2014), Takemoto et al. (2016, } \\
\text { 2017) }\end{array}$ & $\begin{array}{l}\text { Intermittent atrial pacing at cycle length } \\
50 \text { ms for } 30 \text { s duration followed by } 10 \\
\text { s sensing for a range of } 9-24 \text { weeks }\end{array}$ & $\begin{array}{l}\text { AF lasting }>1 \text { week after cessation of } \\
\text { pacing }\end{array}$ & $100 \%$ \\
\hline
\end{tabular}

AF, atrial fibrillation.

own, continuous tachypacing was more successful in producing sustained AF after 15 weeks (77 vs. 29\%). It is therefore possible our low success rate, at least in part, reflects our chosen methodology and either continuous pacing or longer period of intermittent tachypacing may have influenced the number of sheep who went on to develop sustained AF. Whether the use of fibrillation number will show similar predictive power to significantly improve experimental efficiency in other ovine AF models is presently unclear, however, this work highlights the need to critically review and optimise animal models of human pathology wherever possible.

\section{Limitations}

There are a number of limitations which should be considered with regard to the applicability of these results. Firstly, the methodology used in calculating fibrillation number (Equation 2) relied upon a number of assumptions. The dimensions of the atrial tissue were measured using the left atrial anteroposterior diameter calculated on echocardiography, however, it is known this can be a poor estimator of atrial tissue volume (Lester et al., 1999) which is central to the critical mass hypothesis (Byrd et al., 2005; Lee et al., 2013). Unfortunately given limited ultrasound windows available in the majority of sheep, left atrial surface area or volume could be accurately calculated. We therefore used left atrial diameter as it was easily reproducible and has been used in other studies (Hwang et al., 2015). A second limiting factor in calculating fibrillation number was that the wavelength was calculated by performing an electrophysiology study in the right atrium. The left atrium has more relevance to AF seen in humans, particularly as it is the common site where $\mathrm{AF}$ is initiated secondary to rapid firing emanating from the pulmonary veins (Haissaguerre et al., 1998). Small differences in the effective refractory period and conduction velocities between the right and left atria are recognised, where the right atrium can only act as a surrogate marker for left atrial electrophysiology (Li et al., 2001; Prabhu et al., 2017). Unfortunately, we were unable to perform a left atrial study due to access issues across the interatrial septum or via a retrograde aortic approach, therefore small differences in fibrillation number may have arisen using right atrial parameters which alter the predictive power for sustained AF.

A further limitation relates to how we defined sustained atrial fibrillation. We believe our requirement for $15 \mathrm{~min}$ of uninterrupted AF reflects that the sheep have undergone a significant amount of atrial remodelling, such that they show similar pathophysiology to humans with persistent AF [defined as continuous AF lasting longer than 7 days (Hindricks et al., 2020)]. Other groups have typically defined sustained AF by a longer duration of continuous arrhythmia in their large animal models (Willems et al., 2001a; Lenaerts et al., 2009), therefore it is unlikely the low success rate of our protocol is due to an abnormally high threshold for defining sustained AF.

\section{CONCLUSION}

In conclusion, we have shown for the first time that we can predict the likelihood of healthy sheep developing sustained atrial fibrillation after the initiation of an 8-week tachypacing protocol through utilising the critical mass hypothesis. Future research studies involving longstanding AF should consider how the relationship between the dimensions of the atrial tissue and the wavelength can help determine the likelihood of animals reaching arrhythmia endpoints. This has the potential to improve future experimental efficiency by reducing the number of animals required and minimising distress in animals resistant to developing sustained AF.

\section{DATA AVAILABILITY STATEMENT}

The raw data supporting the conclusions of this article will be made available by the authors, without undue reservation.

\section{ETHICS STATEMENT}

The animal study was reviewed and approved by the University of Manchester Animal Welfare and Ethical Review Board. 


\section{AUTHOR CONTRIBUTIONS}

$\mathrm{ND}, \mathrm{AT}$, and KD had the idea for and designed the study. ND, CP, GM, and CS conducted the experiments. ND analysed the data and wrote the manuscript. All authors critically revised the final manuscript and approve of its content.

\section{FUNDING}

$\mathrm{ND}, \mathrm{CP}, \mathrm{CS}, \mathrm{AT}$, and $\mathrm{KD}$ were supported by funding from the British Heart Foundation (FS/17/54/33126; FS/15/28/31476; FS12/34/29565; FS12/57/29717; PG/12/89/29970; and FS/09/002/26487). GM was funded by a Medical Research Council Award 1637974. CP was a clinical lecturer funded by the National Institute for Health Research.

\section{REFERENCES}

Anne, W., Willems, R., Holemans, P., Beckers, F., Roskams, T., Lenaerts, I., et al. (2007). Self-terminating AF depends on electrical remodeling while persistent AF depends on additional structural changes in a rapid atrially paced sheep model. J. Mol. Cell. Cardiol. 43, 148-158. doi: 10.1016/j.yjmcc.2007. 05.010

Benjamin, E. J., Wolf, P. A., D’Agostino, R. B., Silbershatz, H., Kannel, W. B., and Levy, D. (1998). Impact of atrial fibrillation on the risk of death: the framingham heart study. Circulation 98, 946-952. doi: 10.1161/01.cir.98.10.946

Byrd, G. D., Prasad, S. M., Ripplinger, C. M., Cassilly, T. R., Schuessler, R. B., Boineau, J. P., et al. (2005). Importance of geometry and refractory period in sustaining atrial fibrillation: testing the critical mass hypothesis. Circulation 112(9 Suppl), I7-I13.

Denham, N. C., Pearman, C. M., Caldwell, J. L., Madders, G. W. P., Eisner, D. A., Trafford, A. W., et al. (2018). Calcium in the pathophysiology of atrial fibrillation and heart failure. Front. Physiol.. 9:1380.

Deroubaix, E., Folliguet, T., Rucker-Martin, C., Dinanian, S., Boixel, C., Validire, P., et al. (2004). Moderate and chronic hemodynamic overload of sheep atria induces reversible cellular electrophysiologic abnormalities and atrial vulnerability. J. Am. Coll. Cardiol. 44, 1918-1926. doi: 10.1016/j.jacc.2004.07. 055

Filgueiras-Rama, D., Price, N. F., Martins, R. P., Yamazaki, M., Avula, U. M., Kaur, K., et al. (2012). Long-term frequency gradients during persistent atrial fibrillation in sheep are associated with stable sources in the left atrium. Circ. Arrhythm. Electrophysiol. 5, 1160-1167. doi: 10.1161/circep.111.969519

Geddes, L. A., Hinds, M., Babbs, C. F., Tacker, W. A., Schoenlein, W. E., Elabbady, T., et al. (1996). Maintenance of atrial fibrillation in anesthetized and unanesthetized sheep using cholinergic drive. Pacing Clin. Electrophysiol. PACE. 19, 165-175. doi: 10.1111/j.1540-8159.1996.tb03308.x

Haemers, P., Hamdi, H., Guedj, K., Suffee, N., Farahmand, P., Popovic, N., et al. (2017). Atrial fibrillation is associated with the fibrotic remodelling of adipose tissue in the subepicardium of human and sheep atria. Eur. Heart J. 38, 53-61. doi: 10.1093/eurheartj/ehv625

Haissaguerre, M., Jais, P., Shah, D. C., Takahashi, A., Hocini, M., Quiniou, G., et al. (1998). Spontaneous initiation of atrial fibrillation by ectopic beats originating in the pulmonary veins. New Engl. J. Med. 339, 659-666. doi: 10.1056/nejm199809033391003

Heijman, J., Voigt, N., Nattel, S., and Dobrev, D. (2014). Cellular and molecular electrophysiology of atrial fibrillation initiation, maintenance, and progression. Circ. Res. 114, 1483-1499. doi: 10.1161/circresaha.114.302226

Hindricks, G., Potpara, T., Dagres, N., Arbelo, E., Bax, J. J., Blomström-Lundqvist, C., et al. (2020). 2020 ESC guidelines for the diagnosis and management of atrial fibrillation developed in collaboration with the European Association of Cardio-Thoracic Surgery (EACTS). Eur Heart J. 42, 373-498.

Hwang, M., Park, J., Lee, Y. S., Park, J. H., Choi, S. H., Shim, E. B., et al. (2015). Fibrillation number based on wavelength and critical mass in patients who

\section{ACKNOWLEDGMENTS}

We would like to thank Medtronic UK for their donation of equipment to assist with conducting these studies.

\section{SUPPLEMENTARY MATERIAL}

The Supplementary Material for this article can be found online at: https://www.frontiersin.org/articles/10.3389/fphys. 2021.690897/full\#supplementary-material

Supplementary Figure 1 | A typical parasternal long axis (PLAX) view obtained by 2D echocardiography from a sheep. Left atrial $(\mathrm{LA})$ anteroposterior diameter was measured during ventricular systole. Epicardial fat pad thickness (EFT) was measured anterior to the right ventricle using the atrioventricular groove as a reference.

underwent radiofrequency catheter ablation for atrial fibrillation. IEEE Trans. Bio. Med. Eng. 62, 673-679. doi: 10.1109/tbme.2014.2363669

Iacobellis, G., Assael, F., Ribaudo, M. C., Zappaterreno, A., Alessi, G., Di Mario, U., et al. (2003). Epicardial fat from echocardiography: a new method for visceral adipose tissue prediction. Obesity research. 11, 304-310. doi: 10.1038/oby. 2003.45

Kilkenny, C., Browne, W. J., Cuthill, I. C., Emerson, M., and Altman, D. G. (2010). Improving bioscience research reporting: the ARRIVE guidelines for reporting animal research. PLoS Biol. 8:e1000412. doi: 10.1371/journal.pbio.1000412

Kirchhof, P., Benussi, S., Kotecha, D., Ahlsson, A., Atar, D., Casadei, B., et al. (2016). 2016 ESC Guidelines for the management of atrial fibrillation developed in collaboration with EACTS. Eur. Heart J. 37, 2893-2962.

Lee, A. M., Aziz, A., Didesch, J., Clark, K. L., Schuessler, R. B., and Damiano, R. J. Jr. (2013). Importance of atrial surface area and refractory period in sustaining atrial fibrillation: testing the critical mass hypothesis. J. Thor. Cardiovasc. Surg. 146, 593-598. doi: 10.1016/j.jtcvs.2012.04.021

Lenaerts, I., Bito, V., Heinzel, F. R., Driesen, R. B., Holemans, P., D’Hooge, J., et al. (2009). Ultrastructural and functional remodeling of the coupling between $\mathrm{Ca} 2+$ influx and sarcoplasmic reticulum $\mathrm{Ca} 2+$ release in right atrial myocytes from experimental persistent atrial fibrillation. Circ. Res. 105, 876-885. doi: 10.1161/circresaha.109.206276

Lenaerts, I., Driesen, R. B., Hermida, N., Holemans, P., Heidbuchel, H., Janssens, S., et al. (2013). Role of nitric oxide and oxidative stress in a sheep model of persistent atrial fibrillation. Europace 15, 754-760. doi: 10.1093/europace/ eut012

Lester, S. J., Ryan, E. W., Schiller, N. B., and Foster, E. (1999). Best method in clinical practice and in research studies to determine left atrial size. Am. J. Cardiol. 84, 829-832. doi: 10.1016/s0002-9149(99)00446-4

Li, D., Zhang, L., Kneller, J., and Nattel, S. (2001). Potential ionic mechanism for repolarization differences between canine right and left atrium. Circ. Re. 88, 1168-1175. doi: 10.1161/hh1101.091266

Macquaide, N., Tuan, H. T., Hotta, J., Sempels, W., Lenaerts, I., Holemans, P., et al. (2015). Ryanodine receptor cluster fragmentation and redistribution in persistent atrial fibrillation enhance calcium release. Cardiovasc. Res. 108, 387-398. doi: 10.1093/cvr/cvv231

Martins, R. P., Kaur, K., Hwang, E., Ramirez, R. J., Willis, B. C., FilgueirasRama, D., et al. (2014). Dominant frequency increase rate predicts transition from paroxysmal to long-term persistent atrial fibrillation. Circulation 129, 1472-1482. doi: 10.1161/circulationaha.113.004742

Moe, G. K., Rheinboldt, W. C., and Abildskov, J. A. (1964). A computer model of atrial fibrillation. Eur. Heart J. 67, 200-220. doi: 10.1016/0002-8703(64) 90371-0

Monigatti-Tenkorang, J., Jousset, F., Pascale, P., Vesin, J. M., Ruchat, P., Fromer, M., et al. (2014). Intermittent atrial tachycardia promotes repolarization alternans and conduction slowing during rapid rates, and increases susceptibility to atrial fibrillation in a free-behaving sheep model. J. Cardiovasc. Electrophysiol. 25, 418-427. doi: 10.1111/jce.12353 
Nattel, S., Xiong, F., and Aguilar, M. (2017). Demystifying rotors and their place in clinical translation of atrial fibrillation mechanisms. Nat. Rev. Cardiol. 14, 509-520. doi: 10.1038/nrcardio.2017.37

NC3Rs. (2020). Responsibility in the Use of Animals in Bioscience Research: Expectations of the Major Research Councils and Charitable Funding Bodies2019, 3rd Edn. London: NC3Rs.

Pearman, C. M. (2014). An excel-based implementation of the spectral method of action potential alternans analysis. Physiol. Rep. 2:e12194. doi: 10.14814/phy2. 12194

Pearman, C. M., Madders, G. W. P., Radcliffe, E. J., Kirkwood, G. J., Lawless, M., Watkins, A., et al. (2018). Increased vulnerability to atrial fibrillation is associated with increased susceptibility to alternans in old sheep. J. Am. Heart Assoc. 7:e009972.

Prabhu, S., Voskoboinik, A., McLellan, A. J. A., Peck, K. Y., Pathik, B., Nalliah, C. J., et al. (2017). A comparison of the electrophysiologic and electroanatomic characteristics between the right and left atrium in persistent atrial fibrillation: Is the right atrium a window into the left? J. Cardiovasc. Electrophysiol. 28, 1109-1116. doi: $10.1111 /$ jce. 13297

Pruvot, E., Jousset, F., Ruchat, P., Vesin, J. M., Prudat, Y., Zerm, T., et al. (2007). Propagation velocity kinetics and repolarization alternans in a freebehaving sheep model of pacing-induced atrial fibrillation. Europace 9 Suppl 6, vi83-vi88.

Stewart, S., Hart, C. L., Hole, D. J., and McMurray, J. J. (2002). A population-based study of the long-term risks associated with atrial fibrillation: 20-year follow-up of the Renfrew/Paisley study. Am. J. Med. 113, 359-364. doi: 10.1016/s00029343(02)01236-6

Takemoto, Y., Ramirez, R. J., Kaur, K., Salvador-Montanes, O., Ponce-Balbuena, D., Ramos-Mondragon, R., et al. (2017). Eplerenone reduces atrial fibrillation burden without preventing atrial electrical remodeling. J. Am. Coll. Cardiol. 70, 2893-2905. doi: 10.1016/j.jacc.2017.10.014
Takemoto, Y., Ramirez, R. J., Yokokawa, M., Kaur, K., Ponce-Balbuena, D., Sinno, M. C., et al. (2016). Galectin-3 regulates atrial fibrillation remodeling and predicts catheter ablation outcomes. JACC Basic Transl. Sci. 1, 143-154.

Willems, R., Ector, H., Holemans, P., Van De Werf, F., and Heidbuchel, H. (2001a). Effect of different pacing protocols on the induction of atrial fibrillation in a transvenously paced sheep model. Pacing Clin. Electrophysiol. PACE 24, 925-932. doi: 10.1046/j.1460-9592.2001.00925.x

Willems, R., Sipido, K. R., Holemans, P., Ector, H., Van de Werf, F., and Heidbuchel, H. (2001b). Different patterns of angiotensin II and atrial natriuretic peptide secretion in a sheep model of atrial fibrillation. J. Cardiovasc. Electrophysiol. 12, 1387-1392. doi: 10.1046/j.1540-8167.2001.01387.x

Williams, S. E., O’Neill, L., Roney, C. H., Julia, J., Metzner, A., Reissmann, B., et al. (2019). Left atrial effective conducting size predicts atrial fibrillation vulnerability in persistent but not paroxysmal atrial fibrillation. J. Cardiovasc. Electrophysiol. 30, 1416-1427. doi: 10.1111/jce.13990

Wolf, P. A., Abbott, R. D., and Kannel, W. B. (1991). Atrial fibrillation as an independent risk factor for stroke: the framingham study. Stroke 22, 983-988. doi: 10.1161/01.str.22.8.983

Conflict of Interest: The authors declare that the research was conducted in the absence of any commercial or financial relationships that could be construed as a potential conflict of interest.

Copyright (C) 2021 Denham, Pearman, Madders, Smith, Trafford and Dibb. This is an open-access article distributed under the terms of the Creative Commons Attribution License (CC BY). The use, distribution or reproduction in other forums is permitted, provided the original author(s) and the copyright owner(s) are credited and that the original publication in this journal is cited, in accordance with accepted academic practice. No use, distribution or reproduction is permitted which does not comply with these terms. 